Bundesgesundheitsbl - Gesundheitsforsch Gesundheitsschutz 2005 • 48:296-306 DOI 10.1007/s00103-004-1004-3 ๑) Springer Medizin Verlag 2005

\author{
N. Thode ${ }^{1} \cdot$ E. Bergmann ${ }^{2} \cdot$ P. Kamtsiuris ${ }^{2} \cdot$ B.-M. Kurth ${ }^{2}$ \\ ${ }^{1}$ Charité, Berlin \\ ${ }^{2}$ Robert Koch-Institut, Berlin
}

\title{
Einflussfaktoren auf die ambulante Inanspruchnahme in Deutschland
}

\section{Problemstellung}

Mehr als 90\% der Bevölkerung gehen mindestens einmal im Jahr zu einem niedergelassenen Arzt $^{1}$ (ohne Zahnarzt) [1]. Aus einer neueren telefonischen Umfrage des Robert Koch-Institutes (RKI) von 2003 geht hervor, dass in den letzten 3 Monaten $69 \%$ der Frauen und 58\% der Männer einen Arzt aufgesucht haben. ${ }^{2}$ Anders als bei der Inanspruchnahme der stationären Versorgung entscheiden die Patienten in der ambulanten Versorgung in der Regel selbst darüber, ob sie einen Arzt kontaktieren. Individuelle Präferenzen und Motive spielen hier am ehesten eine Rolle [2].

Da bei einem Arztkontakt ggf. eine weitere Versorgung durch das Gesundheitssystem veranlasst wird (Vorsorge, Diagnostik, Therapie, Verordnung von Arznei, Heil- und Hilfsmitteln, Überweisung, Einweisung ins Krankenhaus, Bescheinigung der Arbeitsunfähigkeit, Einleitung von Rehabilitationsmaßnahmen, Begutachtung), nehmen Arztkontakte eine zentrale Stellung für die Inanspruchnahme des gesamten Gesundheitssystems ein und können als Surrogat für ambulante Leistungen angesehen werden. Vor diesem Hintergrund ist die Untersuchung der Einflussgrößen auf die ambulante Kontaktrate von besonderer Bedeutung.

\footnotetext{
${ }^{1}$ Aus Gründen der besseren Lesbarkeit werden im Folgenden die männlichen Bezeichnungen verwendet, womit jedoch immer beide Geschlechter gemeint sind.

${ }^{2}$ Eigene Berechnungen.
}

Die Abhängigkeit der Arztinanspruchnahme vom Bedarf, d. h. vom Gesundheitszustand, erscheint dabei trivial. Aber: Gibt es Faktoren, die über den Bedarf hinaus die Inanspruchnahme beeinflussen, wie z. B. der soziale Status? Sind die Kontaktzahlen insgesamt geringer, wenn $\mathrm{Pa}$ tienten einen Hausarzt haben? Besteht ein Unterschied in der Inanspruchnahme durch gesetzlich und privat Krankenversicherte? Lässt sich die in der Gesundheitsökonomie diskutierte These von der angebotsinduzierten Nachfrage durch einen Einfluss des „Angebots“ an medizinischen Leistungen auf Umfang und Struktur der Inanspruchnahme, z. B. der Arztdichte auf die Kontakthäufigkeit, nachweisen? Und wie äußern sich mögliche Unterschiede in den Kontakthäufigkeiten von Frauen und Männern?

\section{จ Mehr als $\mathbf{9 0 \%}$ der Bevölkerung gehen mindestens einmal im Jahr zu einem niedergelassenen Arzt}

Bivariate Analysen geben auf diese Fragen keine sicheren Antworten, da die Inanspruchnahme von vielen Faktoren gleichzeitig beeinflusst wird. So zeigt sich z. B. für Personen mit niedrigem Sozialschichtindex eine höhere Kontaktrate, wobei allerdings nicht klar ist, ob dieses Ergebnis auf schichtspezifisches Gesundheitsverhalten oder aber einen schlechteren Gesundheitszustand zurückzuführen ist. Ähnliches gilt für alle der oben genannten Fragen, die häufig auch in der Öffentlichkeit diskutiert werden. Im Rahmen des Projektes
„Einflussfaktoren auf die Inanspruchnahme des deutschen Gesundheitswesens und mögliche Steuerungsmechanismen“, das im Schwerpunkt „Versorgungsforschung“ vom Bundesministerium für Bildung und Forschung (BMBF) in Zusammenarbeit mit dem Bundesministerium für Gesundheit und Soziale Sicherung (BMGS) gefördert wurde, kamen diese und weitere Hypothesen auf den Prüfstand der multivariaten Analyse. Die Ergebnisse werden hier vorgestellt.

\section{Verhaltensmodell der ambulanten Inanspruchnahme}

Andersen und seine Mitarbeiter veröffentlichten Ende der 196oer-Jahre ein Verhaltensmodell zur Inanspruchnahme von Gesundheitsleistungen, das seither beständig weiterentwickelt wird $[3,4,5]$. Es deckt ein breites Spektrum möglicher Einflussfaktoren auf die ambulante Inanspruchnahme ab und hat sich international vielfach als Bezugsmodell für die Analyse der Inanspruchnahme bewährt [6, 7]. Die Darstellung des Modells ist in $\bullet$ Abb. 1 wiedergegeben und wird nachfolgend erläutert.

\footnotetext{
${ }^{3}$ "Predisposing Characteristics" lassen sich als "disponierende" oder "prädisponierende Faktoren",,"perceived" und "evaluated Need-Factors" als "selbst wahrgenommene" bzw.,"fremd beurteilte Krankheitsfaktoren“ übersetzen. Für "Enabling-Factors" wird die Übersetzung „erleichternde Faktoren" oder "Zugangsvariablen" vorgeschlagen $[8,9]$. Die Verwendung der englischen Fachbegriffe hat sich in der deutschsprachigen Literatur durchgesetzt.
} 
Im Zentrum des Modells stehen die Kategorien „Predisposing Characteristics“, „Enabling Resources“ und „Need“ als Einflussgrößen auf die Inanspruchnahme von Gesundheitsleistungen. ${ }^{3}$

Unter dem Begriff Predisposing Characteristics fasst Andersen alle Merkmale einer Person zusammen, die sich indirekt auf die Inanspruchnahme auswirken. Die Merkmale werden in die Bereiche demographische Merkmale, Sozialstruktur und Gesundheitseinstellungen aufgeteilt.

Als Need-Faktoren werden die Faktoren bezeichnet, die die Inanspruchnahme als Bedarf im weiteren Sinn direkt beeinflussen. Dabei wird zwischen dem von der betroffenen Person wahrgenommenen Bedarf einerseits und dem durch professionelles Urteil objektivierten Bedarf andererseits unterschieden. Der biologische Bedarf als solcher wird nicht eigens genannt, da er nur vermittelt durch die Wahrnehmung des Arztes oder des Patienten auf die Inanspruchnahme einwirkt.

Die Enabling Resources bezeichnen schließlich die notwendigen Voraussetzungen für die Inanspruchnahme von Gesundheitsleistungen. Es wird zwischen personenbezogenen Ressourcen, wie z. B. dem Vorhandensein einer Krankenversicherung, und gemeindebezogenen Ressourcen, wie z. B. Erreichbarkeit von Versorgungseinrichtungen in einer Gemeinde, unterschieden.

Die Resultate der Inanspruchnahme von Gesundheitsleistungen (Outcome), wie sie sich einerseits im subjektiven und objektiven Gesundheitszustand und andererseits auch in der Zufriedenheit mit der Versorgung zeigen, wirken wiederum auf die Bevölkerung und deren Gesundheitsverhalten zurück. Die Inanspruchnahme findet eingebettet in den gesamtgesellschaftlichen Kontext, insbesondere in das Gesundheitssystem, statt. Die Bereiche Outcome und Environment sind vor allem in länderübergreifenden Studien oder im Zeitvergleich von Bedeutung.

Das Erkenntnisinteresse der vorliegenden Analyse gilt den individuellen und strukturellen Faktoren, die die Inanspruchnahme beeinflussen, und stützt sich damit vorwiegend auf den Modellkern. Anhand der Zusammenstellung von personenbezogenen Daten und Strukturmerkmalen sollen die Faktoren, die repräsentativ für die Bevölkerung Deutschlands die ambu-

Bundesgesundheitsbl - Gesundheitsforsch -Gesundheitsschutz 2005 • 48:296-306 DOI 10.1007/s00103-004-1004-3

c) Springer Medizin Verlag 2005

N. Thode - E. Bergmann - P. Kamtsiuris - B.-M. Kurth

\section{Einflussfaktoren auf die ambulante Inanspruchnahme in Deutschland}

\section{Zusammenfassung}

Der Artikel befasst sich mit der Analyse möglicher Einflüsse auf die Inanspruchnahme niedergelassener Ärzte durch die Bevölkerung in einem Jahr. Die theoretische Grundlage bildet das Verhaltensmodell nach R.M. Andersen, das die Einflussgrößen der Inanspruchnahme in die Komponenten „Predisposing ${ }_{\text {, „,Enabling }}$ " und „Need“ konzeptionell unterteilt. Die Datengrundlage ist eine repräsentative Untersuchung der erwachsenen Wohnbevölkerung in der Bundesrepublik Deutschland (Bundes-Gesundheitssurvey 1998), die um regionale Strukturdaten und aggregierte Daten aus der vertragsärztlichen Abrechnung erweitert wurde. Indikatoren für die ambulante Inanspruchnahme sind die erfragten Anzahlen der Kontakte bei niedergelassenen Ärzten insgesamt, der kontaktierten Fachgruppen, der Kontakte bei Internisten und der Kontakte bei Allgemeinmedizinern in den letzten 12 Monaten. Es zeigen sich neben den erwarteten starken Einflüssen der Need-Faktoren wie Morbidität und gesundheitsbezogener Lebensqualität auch Predisposing-Faktoren wie Region, Stadt/Land, Alter und Geschlecht als signifikante Einflussfaktoren auf die Inanspruchnahme niedergelassener Ärzte. Hinsichtlich der Enabling-Faktoren lässt sich festhalten, dass Personen mit Hausarzt entgegen der erhofften Steuerungsfunktion der Hausärzte insgesamt eine höhere Inanspruchnahme aufweisen. Aus den Ergebnissen werden Empfehlungen zu möglichen Steuerungsansätzen abgeleitet und einige Grenzen der Beeinflussbarkeit des Inanspruchnahmegeschehens aufgezeigt.

\section{Schlüsselwörter}

Gesundheitssurvey · Inanspruchnahme niedergelassener Ärzte · Arztkontakte . Gesundheitsversorgung

\section{Predictors for ambulatory medical care utilization in Germany}

\section{Abstract}

An analysis of the possible influences on primary health care utilization is made. The theoretical framework is the health behavioural model of R.M. Andersen, dividing the influencing variables into the components predisposing characteristics, enabling resources, and need factors. The study was based on data from the German National Health Examination and Interview Survey of the adult population in 1998. The data were linked to regional structural data and aggregated account data. The total number of contacts, the number of contacts with general practitioners and internists, and the number of different specialists contacted in the last 12 months, as calculated from interview data, were used to indicate primary health care utilization. In addition to the expected strong influence of need factors such as morbidity and health-related quality of life, the predisposing characteristics such as region, urban/rural, age, and gender have a significant influence on the primary care utilization. With regard to the enabling factors, persons with a family doctor had more contacts overall, which contrasts with the family doctor's assumed gate-keeper function. From the results of the study, recommendations on possible regulatory measures are given, but also some restrictions for influencing the number of contacts are pointed out.

\section{Keywords}

Health examination survey/

Health interview survey (HES/HIS) .

Health care utilization - Ambulatory medical care - Number of doctor visits - Primary care 


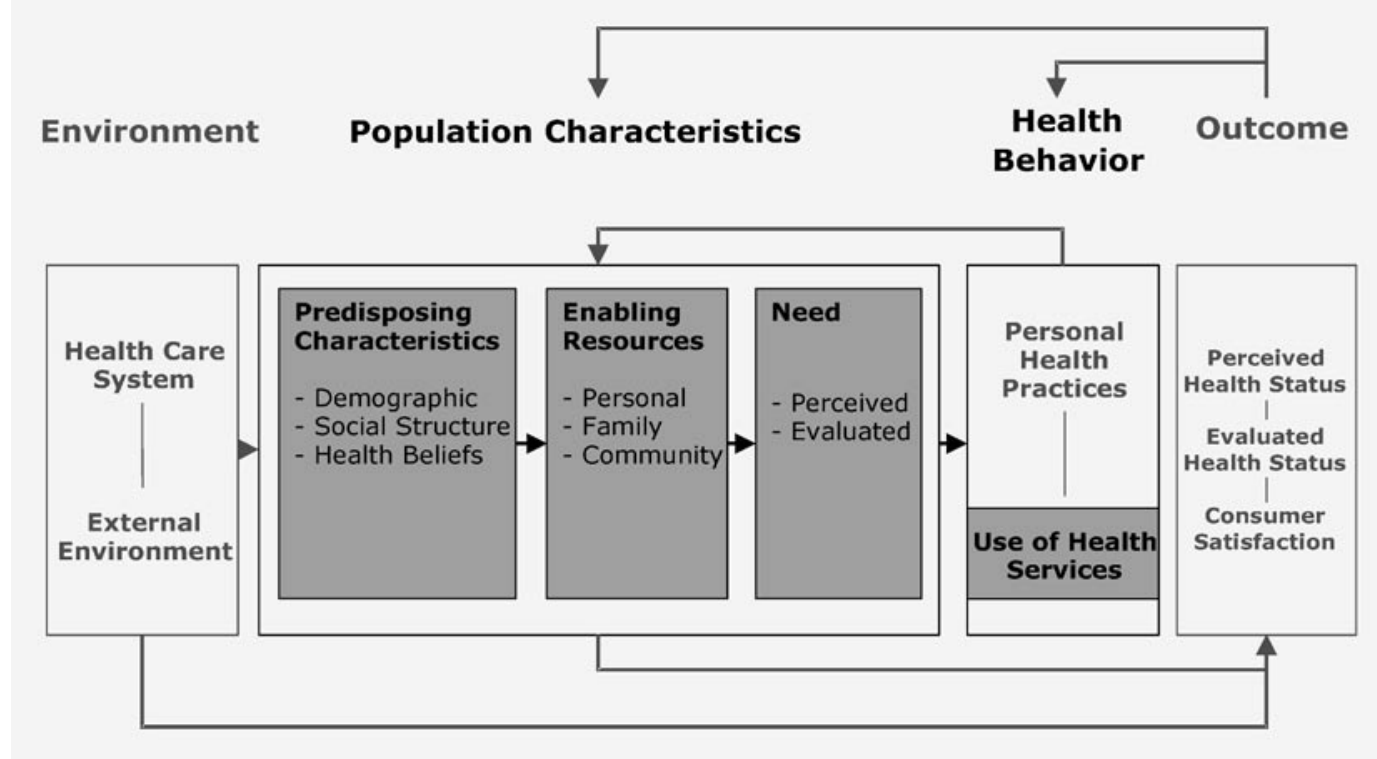

Abb. $1<$ Health Behavior Model nach Andersen [5]

lante Inanspruchnahme erklären, identifiziert werden.

\section{Datenbasis und Methode}

Die Analyse beruht auf den Daten des Bundes-Gesundheitssurveys 1998 (BGS98), der um Informationen aus der vertragsärztlichen Abrechnung und um Strukturmerkmale ergänzt wurde. Der BGS98 ist eine bundesweite, repräsentative Querschnittstudie zur gesundheitlichen Lage der erwachsenen Wohnbevölkerung in Deutschland, die vom RKI im Auftrag des BMGS durchgeführt wurde. Insgesamt wurden 7124 Personen im Alter von 18-79 Jahren befragt und ärztlich untersucht. ${ }^{4}$

Es wurden aus den Selbstangaben der Befragten zur fachgruppenspezifischen Inanspruchnahmehäufigkeit in den letzten 12 Monaten die 4 Zielgrößen „Zahl der Arztkontakte insgesamt “, „Zahl der unterschiedlichen in Anspruch genommenen Fachgruppen“, die „Kontakthäufigkeit bei Allgemeinmedizinern“ und „die Kontakthäufigkeit bei Internisten“ errechnet. Darüber hinaus wurden aus den Angaben der Befragten und den Untersuchungsergebnissen eine Reihe von Merkmalen aus den Bereichen der Predisposing-, Needund Enabling-Faktoren im Hinblick auf ihren Einfluss auf die Inanspruchnahme geprüft. Eine ausführliche Darstellung der Indikatorenbildung findet sich bei [12].

${ }^{4}$ Für eine umfassende Darstellung des BGS98 s. $[10,11]$.
Zur Abbildung des sozialen Status wurde der Sozialschichtindex nach Winkler (SWI) verwendet. In den SWI gehen Angaben zur schulischen und beruflichen Bildung, zur beruflichen Stellung und zum Haushaltseinkommen ein, wobei niedrige Indexwerte mit geringem sozialen Status assoziiert sind [13]. Die in der Analyse verwendeten Skalenwerte liegen zwischen 3 und 21 Punkten.

Das Merkmal Stadt/Land leitet sich aus den BIK-Gemeindetypen ab. Dichotomisiert wird nach den Ausprägungen „Stadt", d. h. der Wohnort der Befragten liegt in einem Oberzentrum mit mehr als 50.00o Einwohnern, und „Land“.

Gesundheitseinstellungen und Gesundheitsbewusstsein, wie beispielsweise gesundheitsbezogene Kontrollüberzeugungen oder Einstellungen zum Versorgungssystem, wurden im BGS98 nicht gesondert abgefragt. Stattdessen werden Angaben zu körperlicher Aktivität, Rauchverhalten und Adipositas als Indikatoren verwendet, die im engen Zusammenhang mit den Gesundheitseinstellungen der Befragten stehen.

Die Gesundheit stellt als mehrdimensionales Konzept hohe Ansprüche an die Operationalisierung. Es wurde versucht, den Gesundheitszustand möglichst umfassend in die Analyse einfließen zu lassen. Dazu wurden 4 Bereiche - gesundheitsbezogene Lebensqualität, Morbidität, akute Ereignisse und psychische Beeinträchtigungen - voneinander abgegrenzt, die jeweils durch mehrere Indikatoren abge- bildet werden. Zusätzlich werden mit der Schwangerschaft bzw. der Schwerbehinderung (als Grad der Behinderung in Prozent) besondere körperliche Umstände berücksichtigt.

Die Morbidität wird durch die Merkmale „Anzahl der Krankheiten im Leben“ und „Anzahl der Krankheiten in den letzten 12 Monaten“ abgebildet. Diese Merkmale beziehen sich auf die im BGS98 erfasste Liste von Krankheiten. Die diesbezüglichen Selbstangaben wurden im Arztinterview validiert und zeitlich eingeordnet. Dabei gilt die Anzahl der Krankheiten im letzten Jahr als direkter Indikator für den Untersuchungszeitraum des BGS98, während die Anzahl der Krankheiten im Leben den allgemeinen Gesundheitszustand einer Person widerspiegelt.

Der SF-36 (Short Form 36 Questionnaire) wird als Instrument zur Messung der subjektiven gesundheitsbezogenen Lebensqualität eingesetzt [14]. Die ursprünglichen 8 Skalen wurden faktoranalytisch zu einem psychischen Faktor und einem körperlichen Faktor zusammengefasst. Hier werden die standardisierten Faktorwerte verwendet, wobei höhere Werte eine bessere subjektive psychische bzw. körperliche Gesundheit abbilden. Zusätzlich wird die Selbsteinschätzung des Gesundheitszustandes auf einer 5-stufigen Skala aus dem Arztinterview verwendet, wobei hohe Werte eine schlechtere Einschätzung angeben.

Befindlichkeitsstörungen und psychische Auffälligkeit werden mit der von 
Tabelle 1

\section{Ergebnisübersicht der Modellierung}

\begin{tabular}{|c|c|c|c|c|c|c|}
\hline & & $\begin{array}{l}\text { Anzahl } \\
\text { Arztkontakte }\end{array}$ & $\begin{array}{l}\text { Anzahl } \\
\text { kontaktierter } \\
\text { Fachgruppen }\end{array}$ & $\begin{array}{l}\text { Anzahl Kontakte } \\
\text { bei Allgemeinme- } \\
\text { dizinern }\end{array}$ & $\begin{array}{l}\text { Internisten } \\
\text { Kontakt-Wahr- } \\
\text { scheinlichkeit }\end{array}$ & $\begin{array}{l}\text { Anzahl der } \\
\text { Kontakte }\end{array}$ \\
\hline $\begin{array}{l}\text { Predis- } \\
\text { posing }\end{array}$ & $\begin{array}{l}\text { - Frauen } \\
\text { - Alter } \\
\text { - Alter - Männer } \\
\text { - Alter - Frauen } \\
\text { - Land } \\
\text { - West } \\
\text { - Erwerbstätigkeit } \\
\text { - Belastungen am Arbeitsplatz } \\
\text { - Andere Nationalität } \\
\text { - Andere Nationalität - Männer } \\
\text { - Andere Nationalität - Frauen } \\
\text { - Höherer Sozialschichtindex } \\
\text { - Gesundheitsverhalten: sportlich aktiv } \\
\text { - Gesundheitsverhalten: Raucher } \\
\text { - Gesundheitsverhalten: Adipositas } \\
\text { - Zusammenleben mit Partner } \\
\text { - Anzahl der Helfer im Notfall } \\
\text { - Allgemeine Lebenszufriedenheit }\end{array}$ & $\begin{array}{l}+ \\
0 \\
0 \\
0 \\
- \\
- \\
-\end{array}$ & $\begin{array}{l}+ \\
0 \\
0 \\
- \\
-\end{array}$ & + & $\begin{array}{l}+ \\
+ \\
+\end{array}$ & + \\
\hline Enabling & $\begin{array}{l}\text {-Hausarzt } \\
\text { - Hausarzt - Männer } \\
\text { • Hausarzt - Frauen } \\
\text { - Privat versichert } \\
\text { - Privat versichert - Männer } \\
\text { - Privat versichert - Frauen } \\
\text { - Allgemeinarztanteil } \\
\text { - Internistenanteil } \\
\text {-Arztdichte } \\
\text { - KV-Abrechnungsmerkmale }\end{array}$ & + & + & $\begin{array}{l}+ \\
+\end{array}$ & + & + \\
\hline Need & $\begin{array}{l}\text { - Anzahl Krankheiten (12 Monate) } \\
\text { - Anzahl Krankheiten (Leben) } \\
\text { - Abwesenheit von Krankheiten } \\
\text { - Selbsteinschätzung des Gesundheitszustands } \\
\text { - Psychischer Faktor (SF-36) } \\
\text { - Körperlicher Faktor (SF-36) } \\
\text { - Verletzungen } \\
\text { - Verletzungen - Männer } \\
\text { - Verletzungen - Frauen } \\
\text { - Anzahl Schmerzlokalisationen } \\
\text { - Anzahl Schmerzlokalisationen - Männer } \\
\text { - Anzahl Schmerzlokalisationen - Frauen } \\
\text { - Grad der Behinderung } \\
\text { - Schwangerschaft } \\
\text { - Anzahl grippale Infekte } \\
\text { - Psychische Auffälligkeit (CID-S) } \\
\text { - Befindlichkeitsstörungen (v. Zerssen BL) } \\
\text { - Ärztlicher Befund }\end{array}$ & $\begin{array}{l}+ \\
+ \\
\text { (entfällt) } \\
+ \\
- \\
-\end{array}$ & $\begin{array}{l}+ \\
+ \\
\text { (entfällt) } \\
+ \\
- \\
- \\
+\end{array}$ & $\begin{array}{l}+ \\
+ \\
\text { (entfällt) } \\
+ \\
- \\
- \\
+\end{array}$ & $\begin{array}{l}\text { (entfällt) } \\
\text { (entfällt) } \\
- \\
+ \\
\text { (entfällt) } \\
\text { (entfällt) }\end{array}$ & $\begin{array}{l}+ \\
+ \\
\text { (entfällt) }\end{array}$ \\
\hline
\end{tabular}

Wirkungsrichtungen der Einflussfaktoren auf die Inanspruchnahme: + mehr IA; - weniger IA; o altersspezifische IA; leere Felder kein Einfluss; (entfällt) entfällt wegen Überspezifikation des Modells, SF-36 Short Form 36 Questionnaire. 


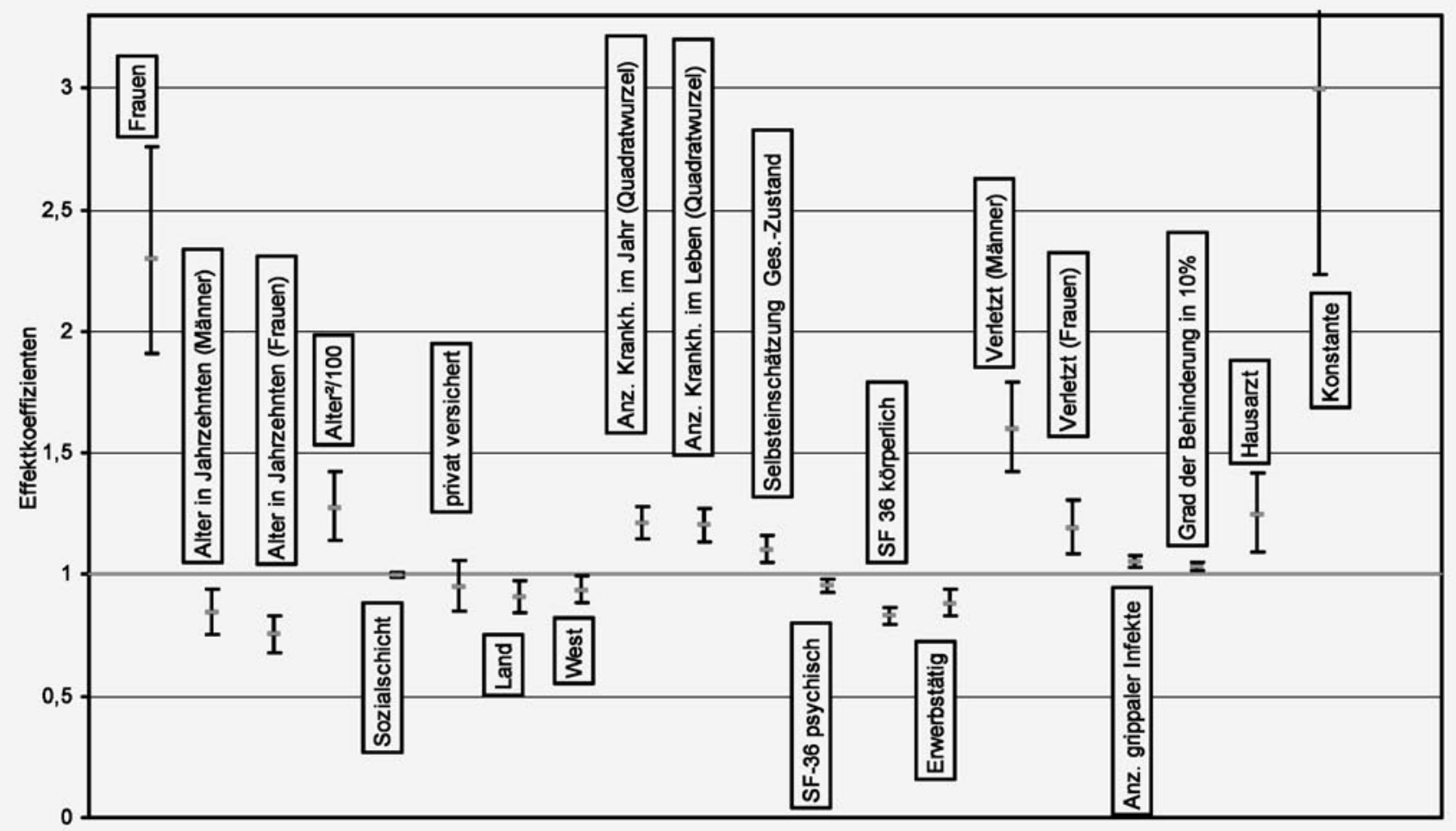

Abb. $2 \Delta$ Multiplikative Effektkoeffizienten für die Anzahl der Arztkontakte insgesamt mit 95\%-Konfidenzintervall. (SF-36 Short Form 36 Questionnaire)

Zerssen Beschwerdenliste (BL) [15] und einer angepassten Form des Composite International Diagnostic Screener (CID-S) [16] durch jeweils eigene Skalen abgebildet.

Um die gemeindebezogenen EnablingFaktoren zu erfassen, wurden neben den Befragungs- und Untersuchungsdaten aus dem BGS98 auch aggregierte Abrechnungsdaten der Kassenärztlichen Bundesvereinigung (KBV) aus dem Jahr 1997 herangezogen. Aus den vorliegenden Routinedaten, jeweils bezogen auf Ärzte in freier Praxis und ergänzt durch eine Sonderauswertung der KBV, wurden die Kontakte pro Fall, der Punktwertverfall und der Umsatz auf KV-Bezirksebene als Indikatoren verwendet. Zusätzlich wurden Daten aus dem Informationssystem „Indikatoren und Karten zur Raumentwicklung “ (INKAR) [17] zur Charakterisierung der Region und zur Beschreibung des Ärzteangebots auf Kreisebene eingebunden. Verwendet wurde die Arztdichte, d. h. die Zahl der Ärzte pro 100.0oo Einwohner, und der Anteil der Ärzte einer bestimmten Fachgruppe an allen Ärzten eines Kreises. Die verschiedenen Datenquellen wurden zur Analyse auf Individualebene zusammengeführt.
Die 4 Zielgrößen Anzahl der Arztkontakte insgesamt, Anzahl der kontaktierten Fachgruppen, Anzahl der Kontakte bei Allgemeinmedizinern und Anzahl der Kontakte bei Internisten wurden jeweils getrennt mittels generalisierter linearer Modelle bzw. Modelle mit Mischverteilungsansatz analysiert. Dazu wurden alle ,unter Verdacht stehenden“ möglichen Einflussfaktoren auf die ambulante Inanspruchnahme (• Tabelle 1$)$ in die Simultanmodelle einbezogen und hinsichtlich ihrer Wirkung untersucht. ${ }^{5}$

\section{Ergebnisse}

\section{Anzahl der Arztkontakte insgesamt}

Die Anzahl der Arztkontakte insgesamt bezogen auf ein Jahr ist eine wichtige Kenngröße für die Steuerung der Inanspruchnahme. Das Modell zur Erklärung der Zahl der Arztkontakte insgesamt wird in - Abb. 2 mit den Effektkoeffizienten dar-

\footnotetext{
${ }^{5}$ Die Vorgehensweise bei der Modellierung und Auswertung ist im Abschlussbericht des Projektes, beziehbar über http://www.rki.de/FORSCH/ FOR2/FORGBE/FORGBE.HTM, dokumentiert.
}

gestellt. Die Effektkoeffizienten sind als Faktoren zu interpretieren, d. h., pro Einheit des erklärenden Merkmals erhöht bzw. verringert sich die Anzahl der geschätzten Kontakte multiplikativ um den entsprechenden Wert. Beispielsweise erhöht sich die Kontaktzahl insgesamt um den Faktor 1,25, wenn man einen Hausarzt hat. Die Fehlerbalken geben das 95\%-Konfidenzintervall der Faktoren an, enthalten diese den Wert 1, so ist dieser Faktor nicht signifikant. Bei der Interpretation der Effektkoeffizienten ist die Skalierung der Ausgangsvariablen zu beachten. Soweit nicht anders angegeben, sind die Ausgangsvariablen als Dummy-Variablen kodiert. Die Konstante geht ebenso wie die Effektkoeffizienten der inhaltlichen Faktoren multiplikativ in die Berechnung der Schätzwerte ein.

Aus der Gruppe der Predisposing-Faktoren erweisen sich das Geschlecht, das Alter, die Region (Ost/West), Stadt/Land, die Erwerbstätigkeit sowie die Wechselwirkung zwischen Geschlecht und Alter als Einflussgrößen für die Zahl der Arztkontakte insgesamt. Frauen, Personen aus verdichteten und verstädterten Kreisen, aus den neuen Bundesländern sowie Nichter- 


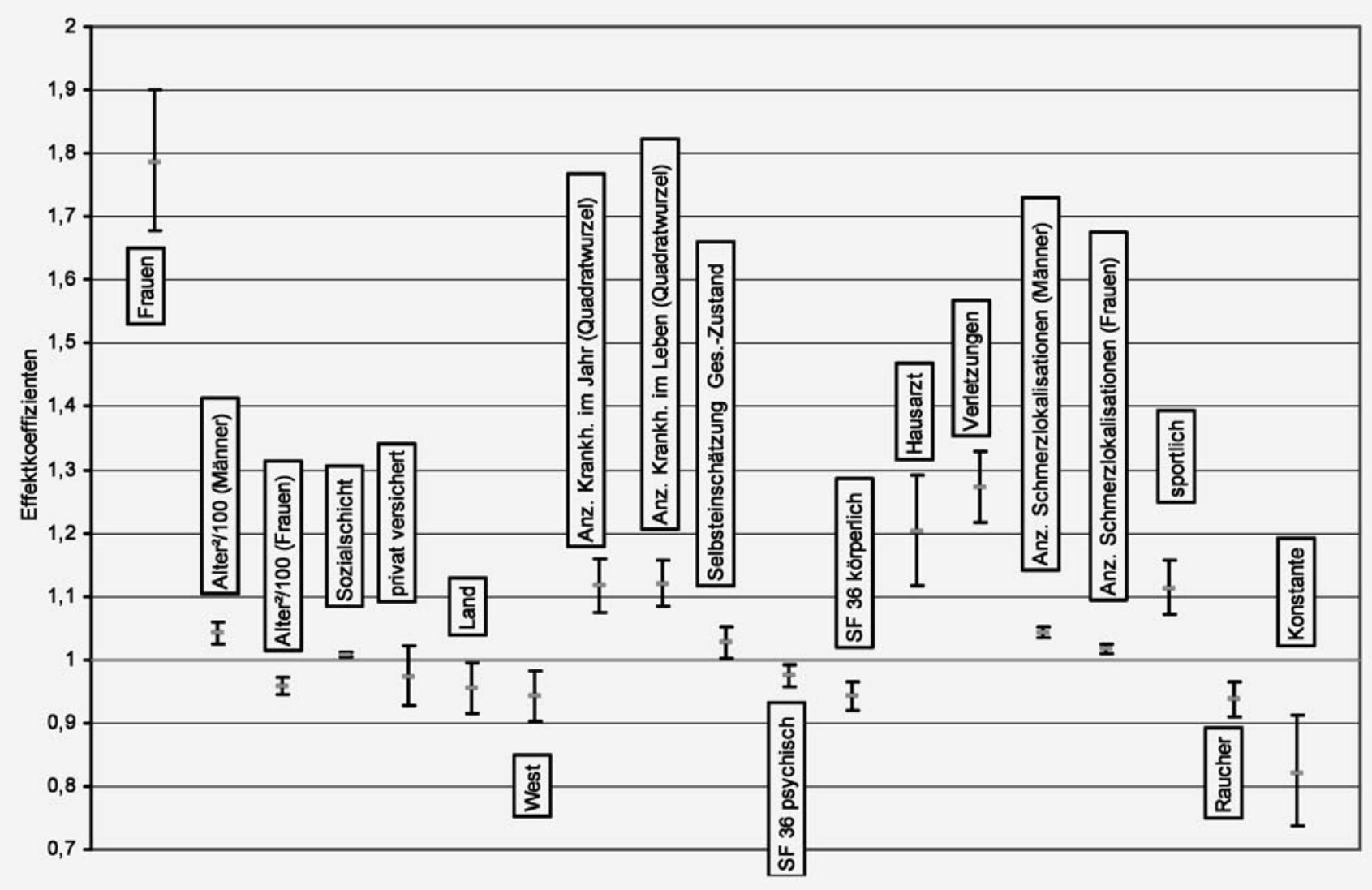

Abb. $3 \Delta$ Multiplikative Effektkoeffizienten für die Anzahl der kontaktierten Fachgruppen mit 95\%-Konfidenzintervall. (SF-36 Short Form 36 Questionnaire)

werbstätige kontaktieren häufiger einen Arzt. Der Einfluss des Alters äußert sich in einer höheren Kontaktzahl von jüngeren Frauen und von älteren Männern, wobei Frauen und Männer im mittleren Alter die geringsten Kontaktzahlen aufweisen. Die in der bivariaten Analyse feststellbare höhere Kontaktrate von Personen mit niedrigem sozialem Status kann hier nicht nachgewiesen werden (vgl. auch - Tabelle 1).

\section{$>$ Personen mit einem Hausarzt zeigen eine höhere ärztliche Inanspruchnahme}

Von den Enabling-Faktoren haben Arztdichte, der Anteil der Allgemeinmediziner an allen Ärzten im Kreis des Wohnorts der Befragten, Abrechnungsmerkmale sowie die Versicherungsart keinen Einfluss auf die Häufigkeit eines Arztbesuches. Allerdings hat der Hausarzt einen Einfluss auf die Kontakthäufigkeit insgesamt: Geben die Probanden an, einen Hausarzt zu haben, so liegt deren Kontaktrate entgegen der oftmals geäußerten Vorstellung höher.

Signifikante Einflussgrößen aus der Gruppe der Need-Faktoren für die Zahl der Arztkontakte sind die Anzahl der Krankheiten in den letzten 12 Monaten und im Leben, die Selbsteinschätzung des Gesundheitszustands, beide Faktoren des SF-36, Verletzungen, die Zahl der Infekte und der Grad der Behinderung. Daneben ist auch die Wechselwirkung von Geschlecht und Verletzungen signifikant, d. h., bei Verletzungen gehen Männer häufiger als Frauen zum Arzt.

\section{Anzahl der kontaktierten Fachgruppen}

Die Anzahl der kontaktierten Fachgruppen gilt als Indikator für die Komplexität der Inanspruchnahme einer Person. Diese ist in Deutschland weitgehend von den Versicherten beeinflussbar, da für diese ein freier Zugang zu niedergelassenen Ärzten aller Fachgebiete besteht. Die Direktinanspruchnahme von Fachärzten wird kritisch diskutiert, weil sie die Koordinie- rungsfunktion des Hausarztes erschwert und zu einer übermäßig aufwändigen Diagnostik und Therapie führen kann [18]. Diese Kritik betrifft die Direktinanspruchnahme einiger Fachgruppen stärker als andere, so gilt beispielsweise die Direktinanspruchnahme von Augenärzten oder Gynäkologen als unproblematisch. Für einen freien Zugang zu Spezialisten spricht, dass der Patient den (Um-)Weg zum Hausarzt spart - unter der Voraussetzung, dass er seinen fachspezifischen Bedarf richtig einschätzt. Dann entfallen auch zusätzliche Leistungen des Hausarztes [19].

Aus der Gruppe der Predisposing-Faktoren besitzen die Variablen Geschlecht, Alter, Region, Stadt/Land, soziale Schicht sowie Risikoverhalten Erklärungsrelevanz: Frauen sowie Personen mit höherem Schichtindex, in stärker verdichteten Kreisen, in den neuen Bundesländern, solche, die regelmäßig Sport treiben und Nichtraucher kontaktieren mehr Fachgruppen. Der vergleichsweise starke Einfluss des Geschlechts ist im Zusammenhang mit der Inanspruchnahme von Gynäkologen zu sehen. Keinen Einfluss auf die Zahl der kon- 


\section{Originalien und Übersichtsarbeiten}

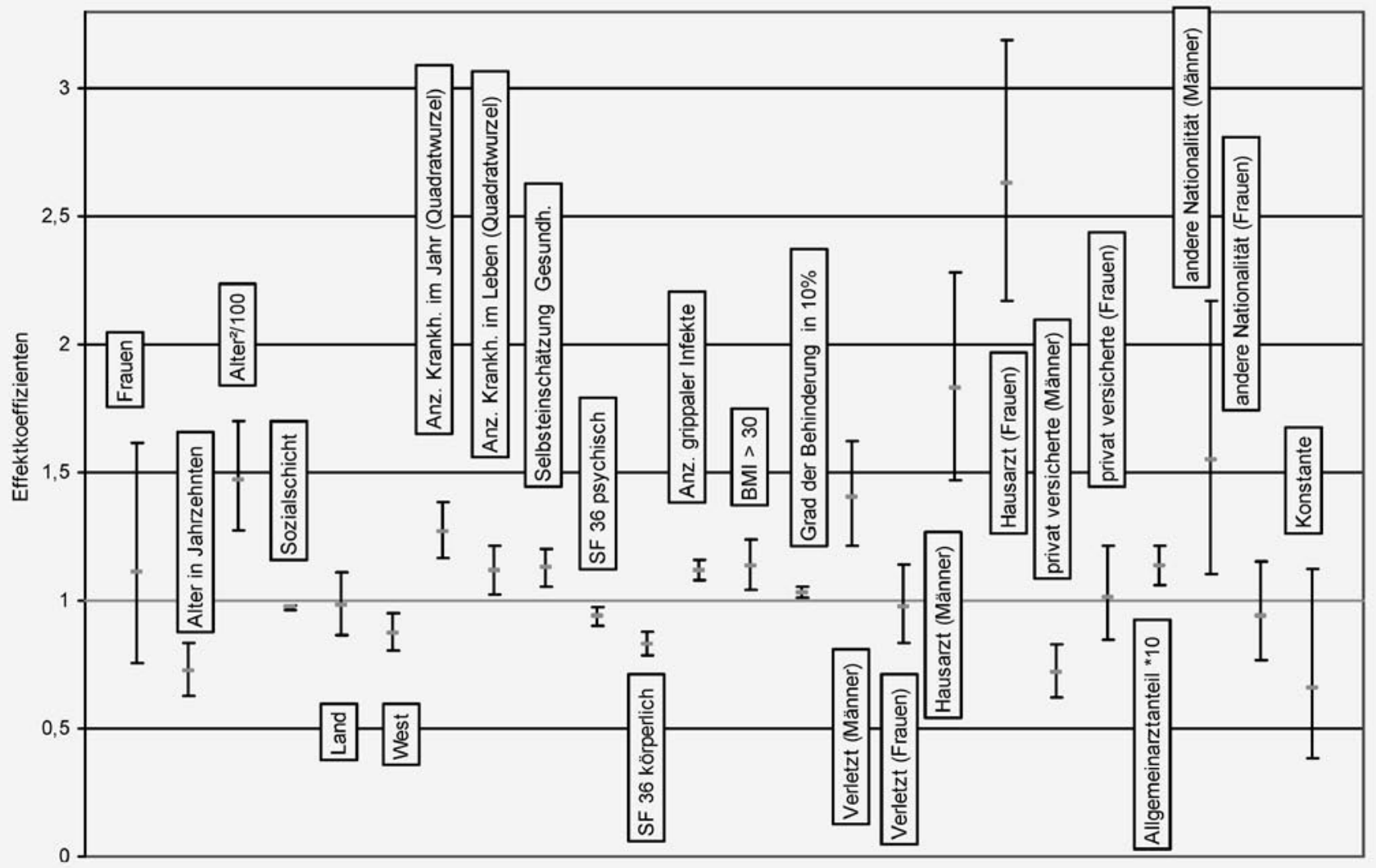

Abb. 4 Multiplikative Effektkoeffizienten für die Anzahl der Arztkontakte bei Allgemeinmedizinern mit 95\%-Konfidenzintervall. (SF-36 Short Form 36 Questionnaire, BMI Body Mass Index)

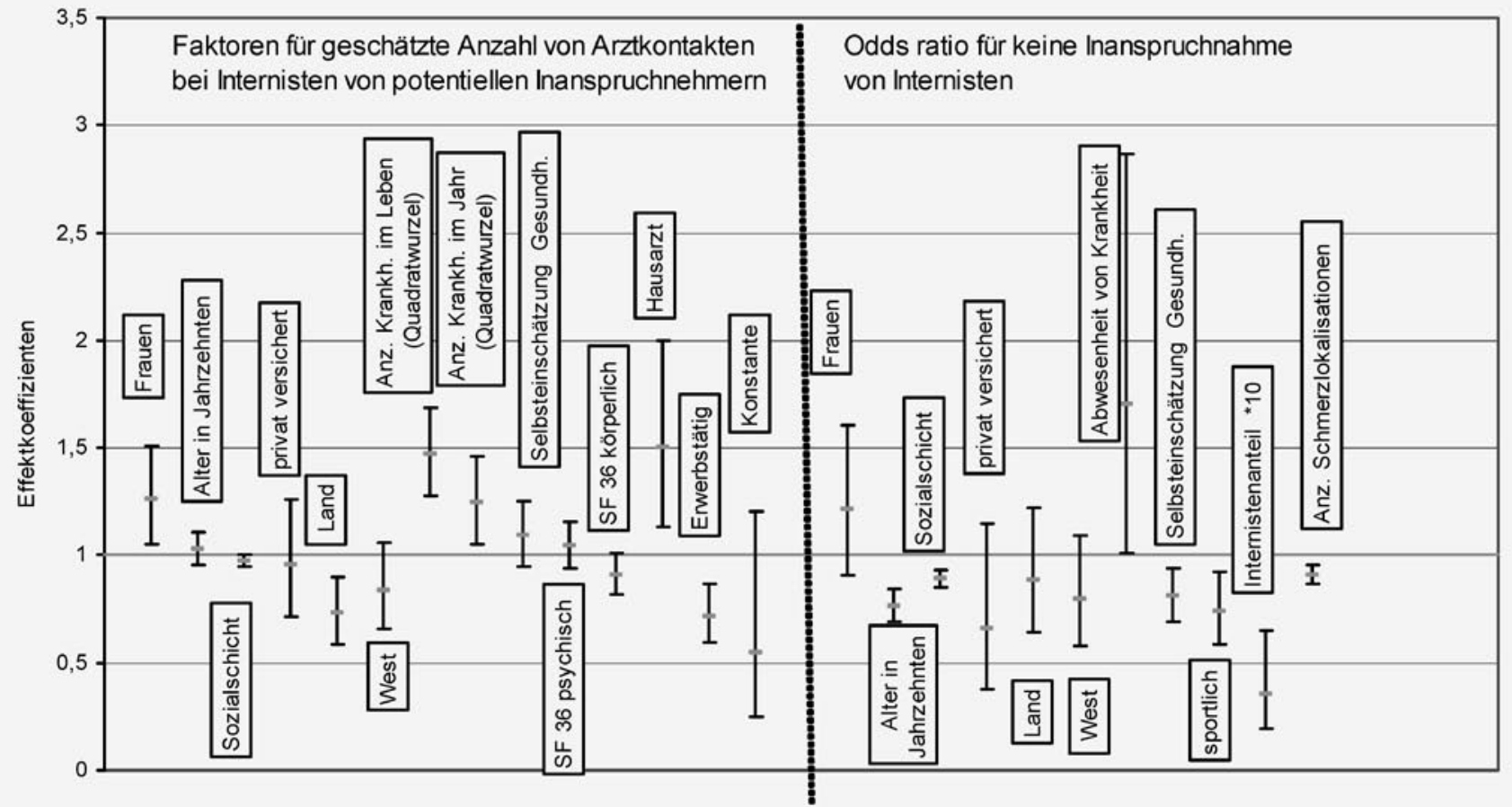

Abb. $5 \Delta$ Multiplikative Effektkoeffizienten für die Kontaktzahl und die Kontaktwahrscheinlichkeit bei Internisten mit 95\%-Konfidenztintervall. (SF-36 Short Form 36 Questionnaire) 
sultierten Fachgruppen haben die Nationalität, die Erwerbstätigkeit, Belastungen am Arbeitsplatz, das Zusammenleben mit einem Partner, die Anzahl der Helfer und die Lebenszufriedenheit.

Für die Enabling-Faktoren Arztdichte, Anteil der Allgemeinmediziner bzw. Internisten an allen Ärzten des Kreises, Abrechnungsmerkmale sowie Versicherungsart kann kein signifikanter Einfluss auf die Zahl der kontaktierten Fachgruppen nachgewiesen werden. Allein das Vorhandensein eines Hausarztes beeinflusst die Zielgröße, wobei auch hier entgegen verbreiteter Vorstellungen Patienten mit Hausarzt mehr Fachgruppen in Anspruch nehmen.

Als wichtigste Einflussgrößen auf die Zahl der kontaktierten Fachgruppen erweisen sich die Need-Faktoren (• Abb. 3). Personen mit mehr Krankheiten sowie schlechterem subjektiven Gesundheitszustand kontaktieren mehr Fachgruppen. Auch Verletzungen und Vergiftungen sowie die Zahl der Schmerzlokalisationen wirken sich auf die Zahl der kontaktierten Fachgruppen aus. Darüber hinaus ist die Wechselwirkung zwischen Geschlecht und der Anzahl der Schmerzlokalisationen signifikant, wobei Männer mit mehr Schmerzen mehr Fachgruppen kontaktieren. Für alle weiteren Need-Faktoren, wie z. B. Befindlichkeitsstörungen und psychische Auffälligkeit, kann kein signifikanter Einfluss auf die Zahl der kontaktierten Fachgruppen nachgewiesen werden.

\section{Anzahl der Arztkontakte bei Allgemeinmedizinern}

Allgemeinmediziner sind die am häufigsten konsultierte Fachgruppe, insgesamt fanden 1998 46\% der Kontakte von Männern und 38\% der Arztkontakte von Frauen mit Allgemeinmedizinern statt. Bezüglich der Kontaktzahl bei Allgemeinmedizinern stellt sich bei den Predisposing-Faktoren das Alter als signifikante Einflussgröße heraus, wobei auch hier Männer und Frauen im mittleren Alter die geringsten Kontaktzahlen aufweisen (• Abb. 4). Darüber hinaus ist die Inanspruchnahme von Allgemeinmedizinern in Westdeutschland geringer. Auch für die Inanspruchnahme eines Allgemeinmediziners kann in der simultanen Analyse ein Zusammenhang zwischen Anzahl der Kontakte und sozialer Schicht, der über die Morbidität hinausgeht, nachgewiesen werden. Personen mit geringerem sozialen Status gehen häufiger zum Allgemeinmediziner.

Es wird ein signifikanter geschlechtsspezifischer Einfluss bei den Merkmalen „Verletzungen/Vergiftungen aufgetreten“, „Hausarzt vorhanden“, „Versicherungsart GKV/privat“ und „Nationalität deutsch/ nicht deutsch“ aufgezeigt. Für Frauen kann, anders als bei Männern, weder für die Verletzungen, noch für die Versicherungsart, noch für die Nationalität ein Einfluss nachgewiesen werden. Demgegenüber ist der Einfluss des Merkmals „Hausarzt vorhanden“ bei Frauen noch stärker ausgeprägt. Ein globaler Einfluss des Geschlechts, der über diese Wechselwirkungen hinausgeht, wird nicht nachgewiesen.

\section{(7) Allgemeinmediziner sind die am häufigsten konsultierte Fachgruppe}

Die Need-Faktoren erweisen sich auch bei dieser Zielgröße der Inanspruchnahme als die wichtigsten Einflussvariablen. Eine höhere Anzahl der Krankheiten in den letzten 12 Monaten und im Leben, eine schlechtere Selbsteinschätzung des Gesundheitszustandes, eine höhere Anzahl von Infekten, Adipositas und das Vorliegen von Verletzungen/Vergiftungen bei Männern führt zu einer höheren Anzahl von Kontakten. Auf der anderen Seite verringert sich die Inanspruchnahme von Allgemeinmedizinern bei guten physischen und psychischen SF-36-Faktorwerten.

Während die Enabling-Faktoren Versicherungsart und Arztdichte sowie Abrechnungsmerkmale keine signifikante Wirkung besitzen, weist der Allgemeinmedizineranteil im Kreis des Wohnortes des Befragten einen signifikant positiven Einfluss auf die Inanspruchnahme dieser Fachgruppe auf.

\section{Anzahl der Kontakte und Inanspruchnahmewahrscheinlich- keit von Internisten}

Internisten waren während des Befragungszeitraums einerseits in der hausärztlichen Versorgung tätig, andererseits erbringen hausärztlich tätige Internisten in nennenswertem Umfang auch fachärztliche
Leistungen (z. B. Diagnostik) und werden entsprechend fachärztlich in Anspruch genommen [20]. Etwas mehr als 20\% der Befragten haben in den letzten 12 Monaten einen Internisten aufgesucht. Die große Anzahl der Personen ohne Kontakt bei Internisten wird in der statistischen Analyse durch ein gemischtes Modell berücksichtigt, in dem die Kontaktwahrscheinlichkeit und die Kontakthäufigkeit simultan geschätzt werden.

In $\bullet$ Abb. 5 werden die Effektkoeffizienten für die Kontaktwahrscheinlichkeit und die Kontakthäufigkeit dargestellt. Im linken Teil sind die Koeffizienten, die für die Zahl der Kontakte ausschlaggebend sind, abgebildet. Sie sind wie in den vorangehenden Modellen als Faktoren interpretierbar. Der rechte Teil zeigt die Koeffizienten für die Wahrscheinlichkeit, keinen Internisten in Anspruch zu nehmen, die als Odds Ratios zu interpretieren sind.

Für die Kontaktzahl sind die Predisposing-Merkmale Geschlecht, Stadt/Land, Erwerbstätigkeit, der Enabling-Faktor Hausarzt sowie die Need-Faktoren Anzahl der Krankheiten im Leben und in den letzten 12 Monaten signifikante Einflussgrößen. Dabei ist die Zahl der Kontakte bei Internisten für Frauen, Personen mit mehr Krankheiten und Personen mit Hausarzt höher, während sie für Personen in ländlichen Kreisen und Erwerbstätige niedriger ist.

Für die Kontaktwahrscheinlichkeit zeigt sich, dass höheres Alter, Zugehörigkeit zu einer höheren sozialen Schicht, eine schlechtere Selbsteinschätzung des Gesundheitszustandes, regelmäßiger Sport, ein höherer Internistenanteil und mehr Schmerzlokalisationen die Wahrscheinlichkeit erhöhen, einen Internisten in Anspruch zu nehmen. Zusätzlich erhöht sich diese Wahrscheinlichkeit, wenn die Befragten mindestens eine Krankheit mit tendenziell chronischem Verlauf angegeben haben.

\section{Diskussion}

Die wichtigsten Einflussfaktoren sind die Need-Faktoren

\section{Morbidität}

Je höher die Anzahl der Krankheiten im Leben und in den letzten 12 Monaten, desto größer ist die Anzahl der jährlichen Arztkontakte. Aufgrund der Überlappung der 
Behandlungsanlässe bei Multimorbidität nimmt mit steigender Anzahl der Krankheiten die Inanspruchnahme etwas weniger stark zu. Die Gesamtkontaktzahlen und die Behandlungen beim Allgemeinmediziner sind erhöht, wenn Verletzungen und Vergiftungen aufgetreten sind, und steigen auch mit der Anzahl grippaler Infekte. Eine Behinderung hat ebenfalls eine höhere Inanspruchnahme zur Folge.

\section{Gesundheitsbezogene Lebensqualität}

Die gesundheitsbezogene Lebensqualität (Selbsteinschätzung der Gesundheit und SF-36) hat einen eigenständigen, von der Morbidität unabhängigen signifikanten Einfluss auf die Anzahl der kontaktierten Fachgruppen, die Zahl der Kontakte bei Allgemeinmedizinern und bei Ärzten insgesamt. Auch die Wahrscheinlichkeit, einen Internisten in Anspruch zu nehmen, wird vom subjektiven Gesundheitszustand beeinflusst, jedoch nicht die Kontaktzahl bei Internisten.

Bei der Unterscheidung von subjektiver körperlicher und psychischer Gesundheit dominiert die körperliche Dimension in ihrem Einfluss sowohl auf die Zahl der Kontakte bei Allgemeinmedizinern und Ärzte insgesamt als auch auf die Zahl der kontaktierten Fachgruppen.

\section{Schmerzlokalisationen}

Zwischen der Zahl der Schmerzlokalisationen und der Kontaktzahl bei Allgemeinmedizinern und Ärzten insgesamt besteht kein signifikanter Zusammenhang. Demgegenüber beeinflusst die Anzahl der Schmerzlokalisationen die Wahrscheinlichkeit, einen Internisten aufzusuchen, sowie die Zahl der kontaktierten Fachgruppen.

\section{Erwartungsgemäß spielen auch die Predisposing-Faktoren eine wichtige Rolle}

\begin{abstract}
Alter
Das Alter erweist sich für alle 4 untersuchten Inanspruchnahmeindikatoren als signifikante Einflussgröße, für die Zahl der kontaktierten Fachgruppen und die Anzahl der Arztkontakte besteht zudem eine Wechselwirkung von Alter und Geschlecht. Der Zusammenhang zwischen Alter und Kontakthäufigkeit mit Ärzten
\end{abstract}

insgesamt als auch mit Allgemeinmedizinern ist U-förmig, d. h. Patienten mittleren Alters haben geringere Kontaktzahlen als jüngere bzw. ältere Patienten. Demgegenüber nimmt die Wahrscheinlichkeit, einen Internisten zu kontaktieren, sowohl bei Männern als auch bei Frauen mit dem Alter stetig zu. Bezüglich der Zahl der kontaktierten Fachgruppen zeigt sich, dass Frauen mit zunehmendem Alter eine geringere Zahl verschiedener Fachgruppen aufsuchen, während ältere Männer mehr Fachgruppen konsultieren.

\section{Geschlecht}

Bei allen 4 Zielgrößen weisen Frauen eine höhere Inanspruchnahme auf, dabei gibt es mehrere Wechselwirkungen zwischen dem Geschlecht und anderen Einflussgrößen. Somit ist auch von geschlechtsspezifischen Wirkungen der unterschiedlichen Einflussfaktoren auf die Inanspruchnahme auszugehen. Zum Beispiel gehen Männer bei Verletzungen oder bei mehr Schmerzlokalisationen verhältnismäßig häufiger zum Arzt als Frauen.

\section{Regionale Faktoren und Erwerbstätigkeit}

Regionale Faktoren (Stadt/Land) und die Erwerbstätigkeit der Probanden bestätigen ihre Wirkung auf die Inanspruchnahme: Erwerbstätige gehen seltener zum Arzt.

\section{(D) Erwerbstätige gehen seltener zum Arzt}

Bewohner ländlicher Gegenden gehen zwar genauso häufig zum Allgemeinmediziner wie Bewohner verdichteter Kreise, sodass von einer ausreichenden Grundversorgung in den Jahren 1997/98 auszugehen ist, sie haben aber weniger Internistenkontakte und suchen weniger Fachgruppen auf. Die Gesamtzahl der Arztkontakte ist auf dem Land geringer.

\section{Sozialer Status und Risikoverhalten}

Der soziale Status und das Risikoverhalten wirken in folgender Weise auf die Inanspruchnahme: Personen mit hohem sozialen Status und Personen mit geringem Risikoverhalten (Nichtraucher bzw. sportlich Aktive) nehmen mit höherer Wahrscheinlichkeit einen Internisten in Anspruch und kontaktieren mehr verschie- dene Fachgruppen. Suchen Personen mit niedrigerem sozialen Status einen Internisten auf, sind ihre Kontaktzahlen genauso hoch wie die der höheren Schicht. Für Allgemeinmediziner ist dagegen die Kontakthäufigkeit von Personen mit geringem sozialen Status erhöht. Die Gesamtzahl der Arztkontakte bei allen niedergelassenen Ärzten ist ceteris paribus weder vom sozialen Status noch vom Risikoverhalten abhängig.

Von größtem gesundheitspolitischen Interesse sind die Einflüsse der Enabling-Faktoren

\section{Versicherungsart}

Die Versicherungsart hat auf die Kontaktzahl und die Kontaktwahrscheinlichkeit keinen Einfluss. Ausnahme bildet für die Männer die Zahl der Kontakte bei Allgemeinmedizinern. Der aufgrund der bivariaten Analyse erwartete negative Zusammenhang zwischen Privatversicherung und Kontaktzahl lässt sich durch die Einbeziehung weiterer Einflussgrößen - vor allem der Need-Faktoren - nicht mehr nachweisen. Bei einer möglichen Integration von GKV und Privatversicherung könnte dies auch Folgen für den Risikostrukturausgleich haben.

\section{Arztdichte/Abrechnungsmerkmale/ Allgemeinarzt-, Internistenanteil}

Für die Arztdichten und KV-Abrechnungsmerkmale konnte keine signifikante Wirkung auf die 4 Inanspruchnahmeindikatoren nachgewiesen werden. Angebotsseitige Faktoren scheinen daher die Höhe und Komplexität der Inanspruchnahme von Ärzten nicht zu beeinflussen.

Es zeigt sich jedoch, dass die Kontaktzahl bei Allgemeinmedizinern mit dem Anteil der Allgemeinmediziner im Kreis steigt und die Wahrscheinlichkeit für eine Inanspruchnahme von Internisten mit dem Anteil der Internisten an der Gesamtzahl der niedergelassenen Ärzte in einer Region zunimmt. Die Zahl der Kontakte bei einem Internisten ist jedoch unabhängig von deren Anteil an allen Ärzten. Diese Ergebnisse könnten zum einen durch die relative Erreichbarkeit einer Arztgruppe in einer Region, aber auch durch eine stärkere gebietsübergreifende Zusammenarbeit und Arbeitsteilung zwischen den Arztgruppen erklärt werden. 


\section{Hausarzt}

Das Vorhandensein eines Hausarztes beeinflusst signifikant die Anzahl der Kontakte bei Allgemeinmedizinern, Internisten und Ärzten insgesamt sowie die Anzahl der kontaktierten Fachgruppen. Allerdings nicht in der erwarteten Richtung: Probanden, die einen Hausarzt haben, den sie bei gesundheitlichen Problemen zuerst aufsuchen, weisen insgesamt eine höhere Inanspruchnahme auf. Die erhoffte Steuerungsfunktion des Hausarztes ist anhand der Daten nicht nachweisbar, sondern wirkt vermutlich eher Kosten treibend. Dies ist umso wichtiger, als 90\% der erwachsenen Bevölkerung einen Hausarzt haben. Allerdings ist davon auszugehen, dass der Hausarzt in den Jahren 1997/1998 nicht die aktuell in der Diskussion befindliche volle Funktion eines „Gatekeepers“ ausübte.

\section{Steuerungsmöglichkeiten für die ambulante Inanspruchnahme}

Die Einteilung der Einflussfaktoren in Predisposing-, Enabling- und Need-Faktoren kennzeichnet von vornherein den Grad bzw. die Möglichkeit der Einflussnahme auf die Inanspruchnahme. Insbesondere die Gruppe der Enabling-Faktoren birgt in sich das Potenzial für Steuerungsansätze zur Reduzierung der Inanspruchnahme medizinischer Leistungen und damit auch letztendlich der Kosten im Gesundheitswesen. Bestehende Vermutungen zur Überinanspruchnahme medizinischer Leistungen durch bestimmte Personengruppen wurden einer systematischen Prüfung unterzogen. Faktoren, die auf einen Missbrauch oder Benachteiligung schließen lassen, konnten dabei nicht gefunden werden. Das Ergebnis, dass es hauptsächlich die NeedFaktoren sind, die die Inanspruchnahme bestimmen, weist auf dreierlei hin.

Erstens sind die Need-Faktoren selbstverständlich keine fixen Größen, sondern vom Gesundheitswesen beeinflussbar. Allgemeinpräventive Maßnahmen, wie z. B. Grippeimpfung und Unfallverhütung, erhalten durch die Ergebnisse der Analyse eine besondere Bedeutung.

Zweitens sind die subjektiven Angaben zur eigenen Gesundheit natürlich stark korreliert mit der subjektiven Einschätzung der Notwendigkeit, medizinische Leistungen in Anspruch zu nehmen. Eine objektivier- bare Einschätzung des Gesundheitszustandes (z. B. durch den jeweils konsultierten Arzt) könnte dieses Dilemma ggf. auflösen. Die Feststellung einer Überinanspruchnahme lässt sich offensichtlich nicht aus den Informationen nur einer Seite des Inanspruchnahmegeschehens, nämlich durch den Patienten selbst, realisieren. Hier erweist sich die Einbeziehung von Krankenkassendaten sicherlich als weitaus effektiver.

Drittens sind die uns zur Verfügung stehenden Informationen zur Inanspruchnahme nicht direkt mit den durch die Inanspruchnahme entstehenden Kosten in Verbindung zu bringen. Auch hier wäre die Verwendung von Krankenkassendaten in Verbindung mit den Surveyinformationen äußerst hilfreich.

Die diskutierten Ansätze zur Steuerung der medizinischen Versorgung, insbesondere der ambulant-ärztlichen Versorgung, auf Individualebene (preisliche Steuerung, Mengensteuerung und Informationssteuerung) haben zum Ziel die Interaktionen zwischen Versicherten/Patienten, Krankenkassen und Leistungserbringer durch die Schaffung von Anreizen effizienter zu gestalten.

\section{(D) Preisliche Steuerungsinstru- mente der Inanspruchnahme von Allgemeinmedizinern besitzen vermutlich keine längerfristige Wirkung}

Bei der Inanspruchnahme der Allgemeinmediziner sind es in erster Linie Need-Faktoren, die die Inanspruchnahme dominieren, was die Annahme einer Preis-unelastischen Nachfrage des Allgemeinmediziners bestätigt. Somit besitzen preisliche Steuerungsinstrumente vermutlich eher keine längerfristige Wirkung. Eine Selbstbeteiligung bei jedem Arztkontakt könnte zwar teilweise extrem hohe Kontaktzahlen reduzieren jedoch um den hohen Preis der Aufhebung des Solidaritätsprinzips zwischen Patienten und gesunden Versicherten.

Demgegenüber ist die Anzahl der Arztkontakte beim Internisten sowie der kontaktierten Fachgruppen auch von den Predisposing-Faktoren Verdichtungsgrad (Stadt/Land als Indikator für die Erreichbarkeit einer Arztpraxis) und Erwerbstätigkeit abhängig und damit von den persönlichen Kosten des Patienten. Dies ist ein Hinweis darauf, dass hier die Preiselastizi- tät der Nachfrage nach medizinischen Leistungen höher liegt. Hier würden preisliche Instrumente eher eine Wirkung haben.

In Bezug auf die Informationssteuerung dürfte der Informationsbedarf bei Versicherten und Patienten der oberen sozialen Schicht und bei gesundheitsbewussten Personen größer sein, sodass, wie unsere $\mathrm{Da}$ ten belegen, die Kontaktwahrscheinlichkeit (jedoch nicht die Kontaktzahl) bei Internisten sowie die Anzahl der kontaktierten Arztgruppen unter gleichen Bedingungen (d. h. auch gleiche Need-Bedingungen) höher sind.

Eine denkbare Strategie, die auch von mehreren Beteiligten des Gesundheitsmarktgeschehens in Erwägung gezogen wird, wäre die Nachfrage- und Angebotsbeeinflussung durch die Reduzierung von Informationsdefiziten sowohl der Versicherten/Patienten als auch der Leistungserbringer. Die Veröffentlichung der Erfolgskennzahlen von Leistungserbringern oder der medizinischen Angebote in elektronischen Medien können eine bessere Information der Patienten bewirken [21]. Ein gut informierter Versicherter kann rationaler entscheiden, welche Leistung er braucht und welche überflüssig sind - d. h. ihm potenziell Schaden zufügen oder zumindest seine Zeit kosten. Hier stellt sich die Frage, ob Krankenkassen selbst dieses Informationsbedürfnis der Versicherten (und Ärzte) befriedigen können. Zum Beispiel ist denkbar, dass die Beratungsfunktion des Medizinischen Dienstes der Krankenversicherung zu Lasten der Kontrollfunktion kostenneutral gesteigert werden kann.

Die Widerlegung bestimmter Vermutungen zur Beeinflussbarkeit des Inanspruchnahmeverhaltens (höhere Angebotsdichte bewirkt auch höhere Inanspruchnahme, Hausarzt verringert die Zahl zusätzlicher Inanspruchnahme, höheres Gesundheitsbewusstsein verringert die Inanspruchnahme, Privatversicherung vermindert entstehende Kosten) erlaubt es aber schon auf der von uns genutzten Datenbasis, mögliche „Sackgassen“ bei Steuerungsansätzen zu meiden.

\section{Korrespondierender Autor \\ Dr. E. Bergmann}

Robert Koch-Institut, Postfach 650261, 13302 Berlin

E-Mail: BergmannE@rki.de 


\section{Literatur}

1. Bergmann E, Kamtsiuris $P$ (1999) Inanspruchnahme medizinischer Leistungen. Gesundheitswesen [Sonderheft 2] 61:138-144

2. Andersen HH, Bormann C, Elkeles T (1993) Kennziffern zur ambulanten Inanspruchnahme. Methodische und inhaltliche Aspekte des Stellenwerts von Surveydaten. Sozial Präventivmedizin 38:26-33

3. Andersen RM (1968) A behavioral model of families' use of health services. In: Research Series. Centerfor Health Ad ministration Studies, The University of Chicago, Chicago

4. Andersen RM, Newman JF (1973) Societal and individual determinants of medical care utilization in the United States. Milbank Quarterly 51:95-124

5. Andersen RM (1995) Revisiting the behavioral model and access to medical care: does it matter? J Health Social Behavior 36:1-10

6. De Boer AGEM, WijkerW, de Haes HCJM (1997) Predictors of health care utilization in the chronically ill: a review of the literature. Health Policy 42:101-115

7. Philips KA, Morrison KR, Andersen RM, Aday LA (1998) Understanding the context of health care utilization: assessing environmental and provider-related variables in the behavioral model of utilization. Health Services Res 33:571-596

8. Härtel U (1985) Soziale Determinanten des Gesundheits- und Krankheitsverhaltens. Ergebnisse und Folgerungen aus der Münchner Blutdruckstudie. HartungGörre, Konstanz

9. Leu RE, Doppmann RJ (1986) Die Nachfrage nach Gesundheit und Gesundheitsleistungen. Ökonomie Gesundheitswesen 159:161-175

10. Bellach BM, Knopf H, Thefeld W (1998) Der Bundes-Gesundheitssurvey 1997/98. Gesundheitswesen 60:59-68

11. Stolzenberg H (2000) Public Use File BGS98. Dokumentation des Datensatzes. Robert Koch-Institut, Berlin

12. Thode N, Bergmann E, Kamtsiuris P, Kurth BM (2004) Einflussfaktoren auf die Inanspruchnahme des deutschen Gesundheitswesens und mögliche Steuerungsmechanismen. http://www.rki.de/FORSCH/FOR2/FORGBE/ WWW_ENDBERICHT.PDF (Stand: 17.3.2004)

13. Winkler J, Stolzenberg H (1999) Der Sozialschichtindex im Bundes-Gesundheitssurvey. Gesundheitswesen 61:178-183

14. Bullinger $M$ (1997) Gesundheitsbezogene Lebensqualität und subjektive Gesundheit. Psychother Psychosom Med Psychol 47:76-91

15. von Zerssen D (1976) Die Beschwerdenliste. Manual. Beltz, Weinheim

16. Wittchen HU, Höfler M, Gander H et al. (1999) Screening for mental disorders: performance of the Composite International Diagnostic-Screener (CID-S). Int J Methods Psychiatric Res 8:59-70

17. Bundesamt für Bauwesen und Raumordnung (1999) Indikatoren und Karten zur Raumentwicklung (INKAR). Eigenverlag, Bonn

18. Sachverständigenrat für die Konzertierte Aktion im Gesundheitswesen (1994) Gesundheitsversorgung und Krankenversicherung 2000. Eigenverantwortung, Subsidiarität und Solidarität bei sich ändernden Rahmenbedingungen. Sachstandsbericht. Baden-Baden

19. Klose J, Schellschmidt H (2001) Finanzierung und Leistungen der Gesetzlichen Krankenversicherung. Einnahmen- und ausgabenbezogene Gestaltungsvorschläge. WIdO Materialien, Bonn

20. Kassenärztliche Bundesvereinigung (2003) Die ärztliche Versorgung in der Bundesrepublik Deutschland zum 31. Dezember 2002. Ergebnisse der Ärztestatistiken der Bundesärztekammer und der Kassenärztlichen Bundesvereinigung. Eigenverlag, Köln

21. Cassel D, Wilke KT (2001) Das Say'sche Gesetz im Gesundheitswesen: Schafft sich das ärztliche Leistungsangebot seine Nachfrage? Eine Analyse zur angebotsinduzierten Nachfrage in der ambulanten ärztlichen Versorgung anhand deutscher Paneldaten. http://www. ub.uni-duisburg.de/ETD-db/theses/available/duett12282001-084213/unrestricted/gesetz.pdf

\title{
Lehrgang zur Vorbereitung auf die Prüfung für den höheren Gesundheitsdienst 2005
}

\author{
Mitteilung der Akademien für Gesundheit, Ernährung und \\ Verbraucherschutz im Bayerischen Landesamt für \\ Gesundheit und Lebensmittelsicherheit vom 9.12.2004,
} Az:: 8042-1/1/04

ie Akademien für Gesundheit, Ernährung und Verbraucherschutz (AGEV) im Bayerischen Landesamt für Gesundheit und Lebensmittelsicherheit (LGL) werden im Jahr 2005 in München einen Lehrgang zur Vorbereitung auf die Prüfung für den höheren Gesundheitsdienst („Amtsarztlehrgang“) abhalten. Die Teilnahme daran ist Voraussetzung für die Zulassung zur Prüfung.

Der Lehrgang gliedert sich in 2 Teile:

1. Lehrgangsteil: 11.4. bis voraussichtlich 29.7.2005,

2. Lehrgangsteil: 5.9. bis voraussichtlich 2.12.2005.

$\mathrm{Zu}$ dem Lehrgang werden maximal 28 Ärztinnen und Ärzte zugelassen werden. Zulassungsgesuche müssen bis spätestens 10. Februar 2005 bei den AGEV im LGL, Veterinärstr. 2, 85762 Oberschleißheim eingegangen sein. Spätere Eingänge können nicht mehr berücksichtigt werden.

Dem Gesuch sind beizufügen:

1. die Approbation als Arzt in Urschrift oder in amtlicher Abschrift,

2. die Promotionsurkunde in Urschrift oder in amtlicher Abschrift,

3. Nachweise über bisherige ärztliche Tätigkeit.

Eine Lehrgangsgebühr wird nicht erhoben.
Bereits bei den Gesundheitsämtern des Freistaates Bayern tätige Ärztinnen und Ärzte brauchen keine Bewerbungsgesuche einzureichen; sie werden zu dem Lehrgang dienstlich entsandt. Bewerber aus den anderen Ländern der Bundesrepublik Deutschland reichen ihre Gesuche über die obersten Landesgesundheitsbehörden ein. Vorrangig werden solche Bewerber zugelassen, die bereits bei einem Gesundheitsamt beschäftig sind.

Richten Sie bitte Ihre Bewerbung an:

Frau Dr. Gerlinde Huber,

Ltd. Veterinärdirektorin,

Bayerisches Landesamt für Gesundheit und Lebensmittelsicherheit, AZ: 8042-1/1-04, 85762 Oberschleißheim 